\title{
Efficacy of Enteral Access in Patients with Esophageal Squamous Cell Carcinoma Under Neoadjuvant Therapy
}

\author{
CHUN-HOU HUANG ${ }^{1,2}$, TSO-FU WANG ${ }^{3,4}$, YI-FENG WU ${ }^{3,4}$, YI-TSO CHENG $^{4,5}$, \\ SHU-FEN LO ${ }^{6}$, TSUNG-CHENG HSIEH ${ }^{1 *}$ and TAI-CHU PENG ${ }^{1,2^{*}}$ \\ ${ }^{1}$ Institute of Medical Sciences, Tzu-Chi University, Hualien, Taiwan, R.O.C.; \\ ${ }^{2}$ Department of Nursing, Tzu-Chi University, Hualien, Taiwan, R.O.C.; \\ ${ }^{3}$ Department of Hematology and Oncology, Buddhist Tzu Chi General Hospital, Hualien, Taiwan, R.O.C.; \\ ${ }^{4}$ School of Medicine, Tzu Chi University, Hualien, Taiwan, R.O.C.; \\ ${ }^{5}$ Department of Cardiovascular Surgery, Buddhist Hualien Tzu Chi Hospital, Hualien, Taiwan, R.O.C.; \\ ${ }^{6}$ Department of Nursing, Tzu Chi University of Science and Technology, Hualien, Taiwan, R.O.C.
}

\begin{abstract}
Background: Enteral feeding tubes used to manage the nutritional status of esophageal cancer were evaluated regarding their effectiveness in patients receiving neoadjuvant therapy. Patients and Methods: A retrospective study evaluating patients with esophageal squamous cell carcinoma undergoing neoadjuvant therapy between 2001 and 2014 was conducted at a medical center. Hospital patients' records for enteral access (EA) insertion and treatment outcomes were statistically analyzed. Results: Patients with EA at initial diagnosis had lower body mass index than the group without $E A(p=0.012)$. Chemotherapy-related adverse events with grade 3-4 mucositis were significantly less frequent in the EA group ( $p=0.008)$, and grade 3-4 anemia was significantly more frequent ( $p=0.012)$. The 4-year mortality rate was $63.0 \%$ in the EA group and $67.7 \%$ in the group without EA ( $p_{n o n-}$ inferiority $=0.035)$, which met the non-inferiority criterion with a non-inferiority margin of 10\%. Conclusion: Patients with esophageal squamous cell carcinoma with malnutrition status undergoing neoadjuvant therapy with EA showed an improved outcome at maintaining nutritional status, reduced severity of mucositis and improved survival rate.
\end{abstract}

*These Authors contributed equally to this study.

Correspondence to: Tai-Chu Peng, Professor, Ph.D., Department of Nursing Institute of Medical Science, Tzu-Chi University, No. 701, Sec. 3, Zhongyang Rd., Hualien 97004, Taiwan, R.O.C. Tel: +886 38565301 ext. 2232, Mobile: +886 975711836, Fax: +886 38574767, e-mail: ptc2008@mail.tcu.edu.tw and Tsung-Cheng Hsieh, Associate Professor, Ph.D., Institute of Medical Sciences, Tzu-Chi University, No. 701, Sec. 3, Zhongyang Rd., Hualien 97004, Taiwan, R.O.C. E-mail: tchsieh@ mail.tcu.edu.tw

Key Words: Enteral access, esophageal squamous cell carcinoma, nutritional status, chemo-radiotherapy, body mass index.
Tri-modality therapy such as neoadjuvant concurrent chemoradiotherapy (CRT) followed by esophagectomy are the primary treatments for locally advanced esophageal cancer (EC). Unfortunately, the 5-year survival rate is rarely greater than $30 \%$ post-treatment (1).

Nutrition plays a critical role in the development and progression of EC. EC patients are subjected to multiple threats leading to malnutrition (2). During multimodality treatment, a patient's nutritional status substantially deteriorates and is associated with significantly poor outcomes (3). In order to reduce the morbidity associated with dysphagia and malnutrition in patients, many centers advocate routine enteral access (EA) placement in all patients undergoing multimodality therapy (4-7). Furthermore, the National Comprehensive Cancer Network and European Society for Medical Oncology guidelines recommend EC treatments implement enteral tubes for feeding $(8,9)$. Recent studies have focused on the routine placement of EA and suggest it may not be a necessary part of neoadjuvant therapy (10-13). CRT has the potential to reduce dysphagia symptoms.

Research into the efficacy of EA for patients with EC during tri-modality therapy has produced inconsistent results. The impact of EA support during CRT in esophageal squamous cell carcinoma (ESCC) remains unclear. Accordingly, the aim of the current study was to retrospectively analyze the effect of EA on nutritional status and clinical outcome in patients with ESCC receiving neoadjuvant treatment.

\section{Patients and Methods}

Patients. This retrospective study was approved by the Research Ethics Committee of the Hualien Tzu Chi General Hospital (no.: IRB-102-21). Patients included in this analysis were (i) who were confirmed as having IA to IIIC ESCC between January 2001 and April 2014 in Hualien Tzu Chi General Hospital; (ii) adequate bone marrow reserve, liver and renal function 1 week prior to initial 
treatment; and (iii) completed radiotherapy and two cycles of chemotherapy regimen with cisplatin combined with continuous infusion of fluorouracil. Patients were excluded if: (i) they had only received surgical, radiotherapy or supportive care; (ii) they had previous history of cancer and concurrent uncontrolled medical illness. The pretreatment clinical stage was based on recordings by imaging, and the TNM staging was based on the American Joint Committee on Cancer Staging Manual (14). Patients were subsequently stratified based on undergoing a procedure to potentially improve nutrition via EA prior to CRT.

Follow-up. Nutritional parameters for both groups (EA, no EA) were collected from the clinical documentation and included: Serum albumin, body mass index (BMI) and body weight (BW), evaluated in both groups at baseline and recorded within 1 week of the first cycle of chemotherapy and again within 1 week of second cycle of chemotherapy. The blood cell count and biochemical data were collected within 1 week post-chemotherapy. Non-hematological adverse events (AEs) such as; nausea, vomiting, diarrhea, mucositis and inflammatory status were assessed during the course of chemotherapy. Adverse events and laboratory abnormalities were graded according to the National Cancer Institute's Common Terminology Criteria for Adverse Events, version 3.0 (15).

Statistical analysis. The data were coded and analyzed using the SPSS statistical package, version 20 (IBM, Armonk, NY, USA). Descriptive statistics such as frequency, percentage, mean, and standard deviation (SD) were provided for the EA and no EA groups. The chi-square test and unpaired $t$-test were performed for comparison between groups for categorical variables and continuous variables, respectively. The non-inferiority test based on FarringtonManning method was conducted for evaluating the effect of EA on 5-year mortality, which was defined from the diagnosis of ESCC until death or the end of December 2014. Considering the 5-year mortality rate difference of $18.3 \%$ between patients with EC with high BMI $\left(>22.5 \mathrm{~kg} / \mathrm{m}^{2}\right)$ and those with low BMI $\left(\leq 22.5 \mathrm{~kg} / \mathrm{m}^{2}\right)$ in a previous study (16), the margin for non-inferiority of EA compared to no EA was set at $10 \%$. Non-inferiority would be claimed if the lower bound of two-sided $90 \%$ confidence interval of the difference of mortality rate between the two groups was less than $10 \%$.

\section{Results}

Patient characteristics. The flowchart presented in Figure 1 outlines this study. A total of 138 patients were assessed for eligibility for inclusion in this study. There were no significant differences between groups regarding most variables. However, the BMI for the EA group was significantly lower than the group without EA $(20.4 \pm 3.5$ vs. $22.2 \pm 3.8, p=0.012)$. No patients received enteral stenting during CRT. The demographics of the study group are shown in Table I.

Nutritional status during chemotherapy. As shown in Figure 2, mean albumin, BMI and BW showed similar reductions during the chemotherapy period in all patients. Despite the worsening of nutritional status during chemotherapy, all patients finished two cycles of chemotherapy and completed radiotherapy. Overall, EA appeared to be associated with

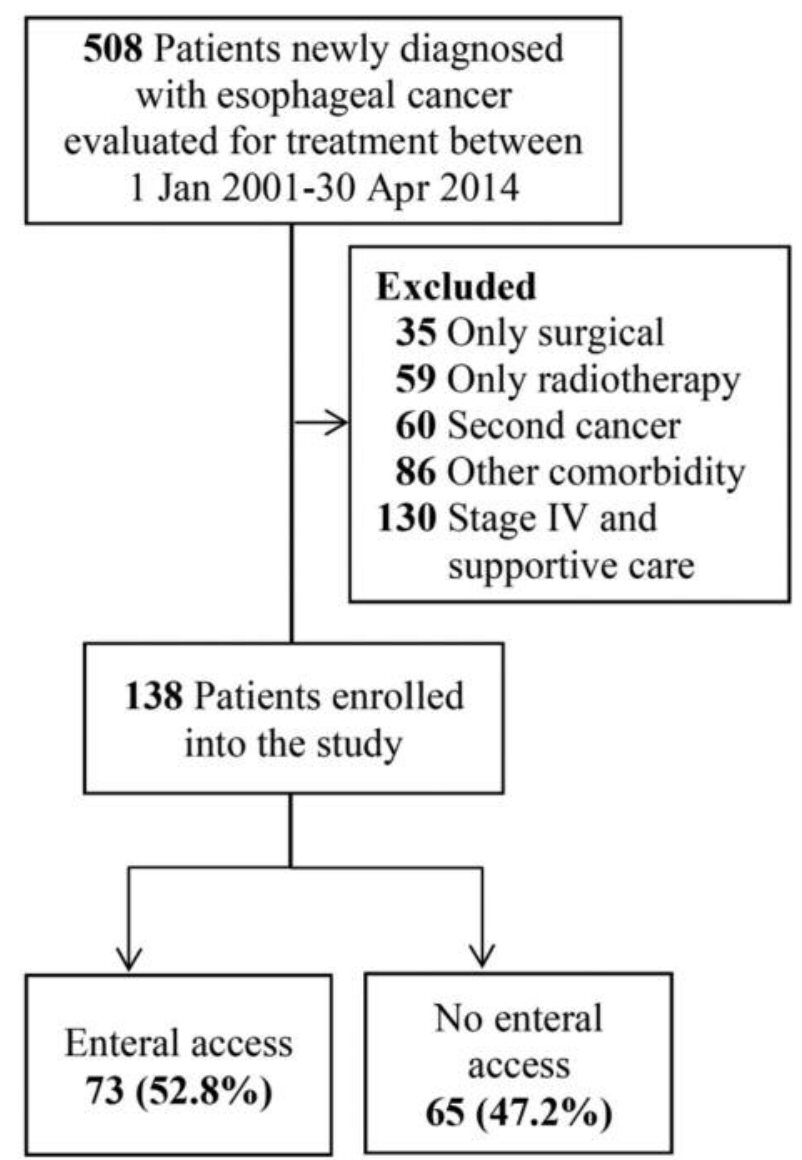

Figure 1. Study flowchart.

maintaining nutritional status among patients who initially presented with a lower BMI and BW.

CRT-related adverse events. AEs during CRT for the two groups can be seen in Table II. Regarding hematological AEs, anemia occurred more often $(5.5 \%)$ in the EA group than in the group without EA (1.5\%). For non-hematological AEs, grade 3 to 4 mucositis was significantly less frequent in the EA group $(p=0.008)$. There were no significant differences noted in the inflammatory status between the two groups.

5-Year mortality rate. The curves for overall mortality for the two groups are shown in Figure 3. In year 3, the EA and no EA groups are virtually the same. In year 4 and 5 , the trend reverses and the no EA group shows a greater mortality compared to the EA group. The 1-year, 2-year and 3-year mortality rates were $38.4 \%, 53.4 \%$, and $58.9 \%$, respectively, in the EA group and $27.7 \%, 47.7 \%$, and 60.0 $\%$, respectively, in the no EA group. Of note, after 3 years, the cumulative mortality rate for the EA group fell below 
Table I. Baseline characteristics for patients with esophageal squamous cell carcinoma $(N=138)$ stratified by use of enteral access (EA).

\begin{tabular}{|c|c|c|c|c|}
\hline Variable & Total & EA & No EA & $p$-Value \\
\hline Number & 138 & 73 & 65 & \\
\hline \multicolumn{5}{|l|}{ Age, years } \\
\hline Mean \pm SD & $53.9 \pm 8.0$ & $51 \pm 7.4$ & $54 \pm 8.5$ & 0.284 \\
\hline \multicolumn{5}{|l|}{ Gender, n (\%) } \\
\hline Male & $130(94.2)$ & $70(95.9)$ & $60(92.3)$ & 0.475 \\
\hline Female & $8(5.8)$ & $3(4.1)$ & $5(7.7)$ & \\
\hline \multicolumn{5}{|l|}{ Alcohol, n (\%) } \\
\hline Never drinker & $1(0.7)$ & $0(0)$ & $1(1.5)$ & 0.473 \\
\hline Ever drinker & $137(99.3)$ & $7(100.0)$ & $64(98.5)$ & \\
\hline \multicolumn{5}{|l|}{ Smoking, n (\%) } \\
\hline Never drinker & $1(0.7)$ & $0(0)$ & $1(1.5)$ & 0.473 \\
\hline Ever drinker & $137(99.3)$ & $73(100.0)$ & $64(98.5)$ & \\
\hline \multicolumn{5}{|l|}{ Betel nut, n (\%) } \\
\hline Never chewing & $12(8.7)$ & $6(8.2)$ & $6(9.2)$ & $>0.999$ \\
\hline Ever chewing & $126(91.3)$ & $67(91.8)$ & $59(90.8)$ & \\
\hline \multicolumn{5}{|l|}{ BMI, $\mathrm{kg} / \mathrm{m}^{2}$} \\
\hline Mean \pm SD & $21.5 \pm 3.8$ & $20.4 \pm 3.5$ & $22.2 \pm 4.1$ & 0.012 \\
\hline \multicolumn{5}{|l|}{ Albumin g/dl } \\
\hline Mean \pm SD & $3.4 \pm 0.5$ & $3.4 \pm 0.5$ & $3.4 \pm 0.5$ & 0.763 \\
\hline \multicolumn{5}{|l|}{ Tumor site, n (\%) } \\
\hline Upper & $20(14.5)$ & $11(15.1)$ & $9(13.8)$ & 0.961 \\
\hline Middle & $58(42.0)$ & $30(41.1)$ & $28(43.1)$ & \\
\hline Lower & $32(23.2)$ & $16(21.9)$ & $16(24.6)$ & \\
\hline Overlapping & $28(20.3)$ & $15(21.9)$ & $13(18.5)$ & \\
\hline \multicolumn{5}{|l|}{ Stage, n (\%) } \\
\hline I & $3(2.2)$ & 0 & $3(4.6)$ & 0.104 \\
\hline II & $44(31.9)$ & $21(28.8)$ & $23(35.4)$ & \\
\hline III & $91(65.9)$ & $52(71.2)$ & $39(60.0)$ & \\
\hline \multicolumn{5}{|c|}{ Esophagectomy, n (\%) } \\
\hline Yes & $69(50.0)$ & $35(47.9)$ & $34(52.3)$ & 0.609 \\
\hline No & $69(50.0)$ & $38(52.1)$ & $31(47.7)$ & \\
\hline \multicolumn{5}{|l|}{ Type of EA } \\
\hline Gastrostomy tube & & 35 & & N/A \\
\hline Jejunostomy tube & & 38 & & \\
\hline
\end{tabular}

BMI: Body mass index, N/A: not applicable. Statistically significant values are shown in bold.

that of the group without EA, with a mortality rate difference of $1.1 \%$ (90\% confidence interval=14.8-12.6\%). The upper boundary at 4 years of $8.7 \%$ was below the non-inferiority margin of $10 \%$; therefore, non-inferiority of EA was established $\left(p_{\text {non inferiority }}=0.035\right.$, Table III).

\section{Discussion}

Malnutrition is a key prognostic factor which impacts survival in EC (17). As a result, EA is a standing recommendation to prevent $\mathrm{BW}$ loss and esophageal obstruction in patients undergoing CRT and surgery (9). The present study suggests that EA is effective at maintaining nutritional status during CRT and reduces mortality in patients with esophageal cancer. Additionally, similar clinical outcomes and toxicities to CRT were found for both groups with and without EA. In patients with EC, it has been reported that $60-80 \%$ present with malnutrition at the time of diagnosis (18). In the present study, patients with EA had significantly lower BMI at baseline than those without EA. Although this finding is subject to selection bias, a similar finding was made by Huerter et al. (11). Their report on 127 patients with EC analyzed nutritional status with EA $(61 / 127)$ or no EA $(66 / 127)$ prior to neoadjuvant therapy. The study showed that EA was associated with improved nutrition in CRT in patients who initially presented as malnourished. By contrast, other studies have shown EA had no impact on BW and albumin during CRT or postoperatively $(13,19,20)$. Furthermore, earlier studies found improvement in dysphagia post neoadjuvant treatment in patients with EC and it was possible to preserve nutrition without EA $(12,21)$. The findings in this study are strongly influenced by the demographics and may not be readily compared with the research of other investigators $(13,20$ - 
$\mathbf{A}$
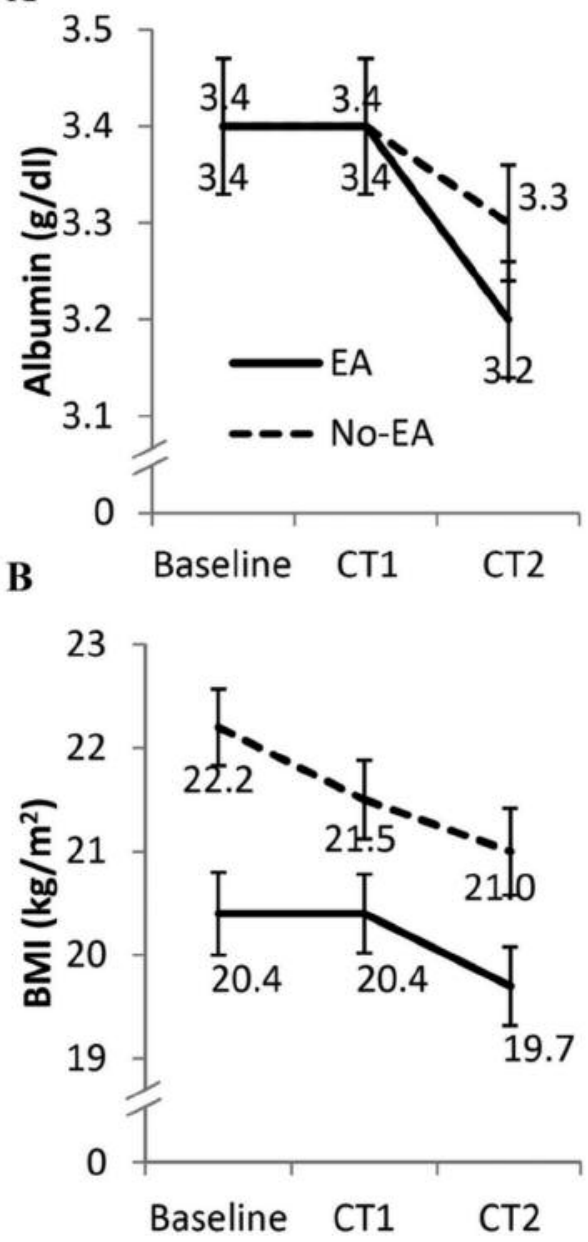

C

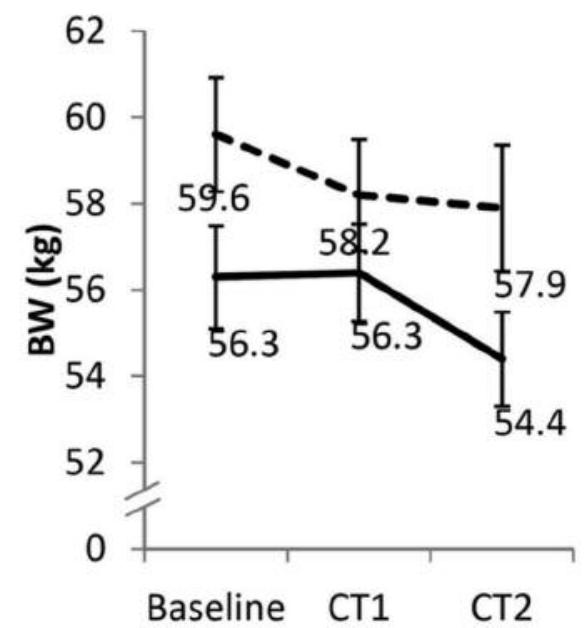

Figure 2. Serum albumin (A), body mass index (BMI) (B) and body weight $(B W)(C)$ during and after neoadjuvant chemoradiotherapy between patients with and without enteral access (EA). Data are the mean ( \pm standard error). There was no statistical difference between the two groups in the data values for any parameter from baseline to the time patients had finished two cycles of chemotherapy (CT).

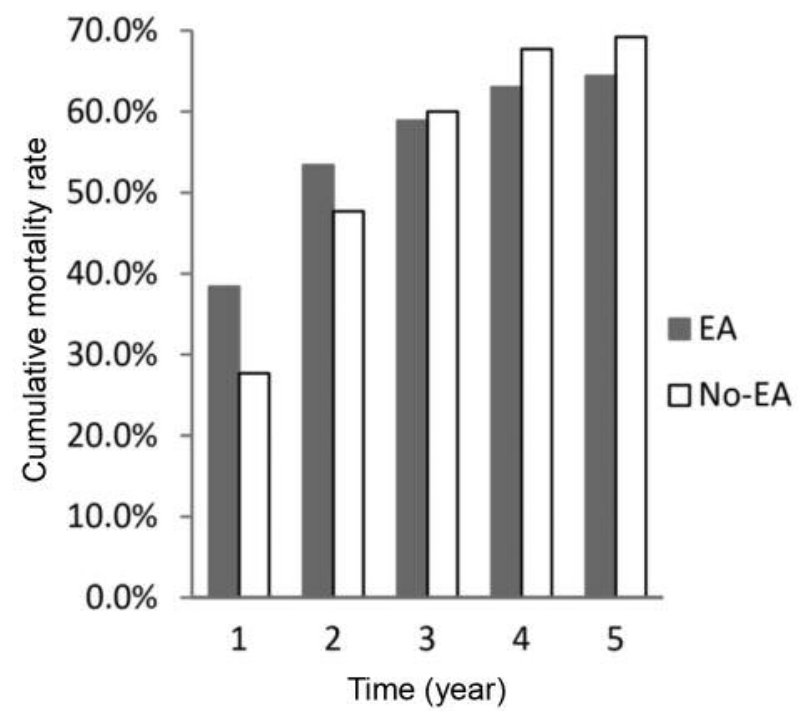

Figure 3. Comparison of accumulation mortality rate for patients with esophageal squamous cell carcinoma undergoing neoadjuvant therapy for malignancy stratified by receipt of enteral access (EA).

22). It must be noted that in these studies, the compared groups had similar levels for nutritional parameters. The phenomenon reflects the question as to whether patients with normal nutritional status require EA prior to neoadjuvant therapy, despite presenting with dysphagia.

In addition, unlike the North American population, squamous cell carcinoma accounts for the majority of EC $(>90 \%)$ in Taiwan (23), and all of our study patients had squamous cell carcinoma. In Western Europe and North America, where being overweight (BMI $\geq 25 \mathrm{~kg} / \mathrm{m}^{2}$ ), or obese (BMI $\geq 30 \mathrm{~kg} / \mathrm{m}^{2}$ ) are more common (24), studies found that BMI had no impact on the outcome of postoperative EC $(25,26)$. In the present study, the EA group had a lower BMI than the group without EA. This suggests a potential benefit with EA for such malnourished patients and may affect the clinical outcome in a positive manner.

Seven out of 63 patients (9.6\%) in the EA group experienced grade 3-4 mucositis during CRT. This is considerably lower than previously published treatment modality which combined platinum-based chemotherapy and radiotherapy, with an associated incidence of $64 \%$ for grade 3-4 oral mucositis (27), which was reduced by up to $10 \%$ through EA support. Radiation therapy induced nearly half the grade 3-4 gastrointestinal mucositis AEs (28), which complicated dysphagia in $15-28 \%$ of patients further necessitating invasive nutritional support (29). The consumption of cigarettes, alcohol, and betel nuts are strongly associated with $\operatorname{ESCC}(23,24)$. Not only low BMI, but history of smoking and beteI nut chewing were risks 
Table II. Adverse events during chemoradiotherapy.

\begin{tabular}{|c|c|c|c|c|c|c|c|c|}
\hline & \multirow[b]{2}{*}{ Adverse event* } & \multicolumn{3}{|c|}{ EA } & \multicolumn{3}{|c|}{ No EA } & \multirow[b]{2}{*}{$p$-Value } \\
\hline & & Grade 0 & Grade 1-2 & Grade $\geq 3$ & Grade 0 & Grade $1-2$ & Grade $\geq 3$ & \\
\hline \multirow[t]{4}{*}{ Hematological } & Leukopenia & $25(34.2)$ & $19(26.0)$ & $29(39.7)$ & $24(36.9)$ & $17(26.2)$ & $24(36.9)$ & 0.932 \\
\hline & Neutropenia & $48(65.8)$ & $15(20.5)$ & $10(13.7)$ & $46(70.8)$ & $8(12.3)$ & $11(16.9)$ & 0.414 \\
\hline & Anemia & $59(80.8)$ & $10(13.7)$ & $4(5.5)$ & $63(96.9)$ & $1(1.5)$ & $1(1.5)$ & 0.012 \\
\hline & Thrombocytopenia & $72(98.6)$ & $1(1.4)$ & & $64(98.6)$ & $1(1.4)$ & & 0.934 \\
\hline \multirow[t]{4}{*}{ Non-hematological } & AST/ALT increase & $70(95.9)$ & $1(1.4)$ & $2(2.7)$ & $62(95.4)$ & $2(3.1)$ & $1(1.5)$ & 0.708 \\
\hline & Creatinine increase & $72(98.6)$ & $1(1.4)$ & & $64(98.6)$ & $1(1.4)$ & & 0.934 \\
\hline & Mucositis & $50(68.5)$ & $16(21.9)$ & $7(9.6)$ & $29(44.6)$ & $19(29.2)$ & $17(26.2)$ & 0.008 \\
\hline & Diarrhea & $65(89.0)$ & $5(6.8)$ & $3(4.1)$ & $60(92.3)$ & $3(4.6)$ & $2(3.1)$ & 0.803 \\
\hline \multirow[t]{3}{*}{ Inflammatory status } & Neutropenic fever & $59(80.8)$ & $2(2.7)$ & $12(16.4)$ & $56(86.2)$ & $1(1.5)$ & $8(12.3)$ & 0.687 \\
\hline & Fever & $50(68.5)$ & $23(31.5)$ & & $48(73.8)$ & $17(26.2)$ & & 0.489 \\
\hline & Infection & $56(76.7)$ & $3(4.1)$ & $14(19.2)$ & $56(86.2)$ & $4(6.2)$ & $5(7.7)$ & 0.138 \\
\hline
\end{tabular}

ALT: Alanine aminotransferase, AST: Aspartate aminotransferase. *By Common Terminology Criteria for Adverse Events, Version 4.02 (15). Statistically significant values are shown in bold.

Table III. Non-inferiority analysis for enteral access (EA) in patients under neoadjuvant therapy for esophageal squamous cell carcinoma.

\begin{tabular}{|c|c|c|c|c|c|c|c|}
\hline \multirow[t]{2}{*}{ Year } & \multicolumn{3}{|c|}{ Mortality rate } & \multicolumn{3}{|c|}{ Non-inferiority test (margin:10\%) } & \multirow[b]{2}{*}{$p$-Value } \\
\hline & EA & No EA & $\begin{array}{l}\text { Rate difference } \\
(\text { EA-no EA) }\end{array}$ & SE & $\begin{array}{l}90 \% \mathrm{CI} \text { of } \\
\text { rate difference }\end{array}$ & $\mathrm{Z}$ & \\
\hline 1 & $38.4 \%$ & $27.7 \%$ & $10.7 \%$ & $7.9 \%$ & $-2.4-23.8$ & 0.083 & 0.533 \\
\hline 2 & $53.4 \%$ & $47.7 \%$ & $5.7 \%$ & $8.4 \%$ & $-8.2-19.6$ & 0.503 & 0.307 \\
\hline 3 & $58.9 \%$ & $60.0 \%$ & $-1.1 \%$ & $8.3 \%$ & $-14.8-12.6$ & 1.328 & 0.092 \\
\hline 4 & $63.0 \%$ & $67.7 \%$ & $-4.7 \%$ & $8.1 \%$ & $-18.0-8.7$ & 1.804 & 0.035 \\
\hline 5 & $64.4 \%$ & $69.2 \%$ & $-4.8 \%$ & $8.0 \%$ & $-18.1-8.4$ & 1.842 & 0.032 \\
\hline
\end{tabular}

CI: Confidence interval, SE: standard error, Z: Z score. Statistically significant values are shown in bold.

factors for the development of moderate to severe oral mucositis during CRT (27). The current findings showed a positive association between EA support during CRT and a reduced incidence of CRT-related mucositis. Based on the current findings, it appears that EA intervention during CRT may reduce the occurrence of mucositis, which is of clinical importance in the treatment of ESCC.

Hematological toxicities were a common occurrence in patients with EC receiving more powerful neoadjuvant treatment (cisplatin-based). Anemia due to bone marrow suppression, along with leukopenia and neutropenia become more common, while the response rate increases (30). The current study showed that EA support during CRT for EC significantly increased grade 3-4 anemia in this study. This may be due to the reduction of normal peptic action mechanisms and defects of absorption of vitamin B12, copper or iron post gastrointestinal surgery (31). The incidence of anemia in the EA group may also be related to their pre-treatment and initial poor nutritional status. One random control study of 91 patients with EC compared EA (47/91) and parenteral nutritional (44/91) for a standard nutritional support formula with omega-3 fatty acid prior to neoadjuvant chemotherapy-related toxicities. The results showed EA support reduced the incidence of chemotherapyrelated leukopenia and neutropenia (20). The benefits of immuno-nutrition have been addressed in meta-analyses (32-34). In the present study, the EA group received adequate nutrition with an appropriate balance of carbohydrates, protein, and lipids. The formulae were not enriched with immuno-nutrients. Our study result remains reasonable as at baseline the BMI was lower, which could progress due to immunosuppression from CRT and become a critical issue in CRT that affects anemia. However, deeper investigations into the effects of severe anemia during CRT in EA patients are necessary in order to fully understand this negative effect. 
One recent study reported compared nutritional parameters changing and perioperative complications in patients with EC with EA (50/99) and without EA (49/99) after multimodality treatment (13). They concluded that there was no nutritional or perioperative benefit of EA before CRT for patients with EC.

Unfortunately, other studies examining EA had small sample sizes, and less robust results with respect to survival or chemotherapy-related toxicity $(11,13,19,20)$. Presently, there is a paucity of research available on the clinical efficacy of EA support during CRT due to differences in the enteral formulas and in the enteral feeding tube such as gastrostomy, jejunostomy and various esophageal stents used in nutritional therapy. Many previous studies investigated whether nutritional support in patients with cancer undergoing chemotherapy or radiotherapy affects tumor response or patient survival. The majority of those studies did not show a positive effect for nutritional support on survival $(11,19,20)$. However, regarding the effect of EA support on patient survival, our findings differ from the literature with respect to survival. Previous research suggests that EA support during CRT was not associated with survival $(11,13,21)$. However, those studies lack of survival differences between the two groups may be because the initial nutritional status levels were normal and similar. By contrast, the current study found that the mortality rate of those in the EA group with low BMI at baseline was not inferior to that of the group without EA with a normal BMI. Therefore, the non-inferiority of EA was established at the 4-year time point. The cumulative mortality rate in the first 3 years was higher in the EA group, but was not statistically significant. In the fourth year, the mortality rate of the EA group became non-inferior to that of the group without EA.

A recent study by Miyazaki et al. revealed that a low BMI $\left(<22.5 \mathrm{~kg} / \mathrm{m}^{2}\right)$ was an independent prognostic indicator for poor survival of ESCC (16). In our study, the patients with lower BMI were considered to have a lower nutritional status. However, the EA group had similar overall survival to patients with normal nutritional status and no EA. The clinical application of these findings is to improve the outcome of CRT-related mucositis and overall survival.

The study also has certain limitations. This study was retrospective. Additionally, there was selection bias regarding the EA group, as use of EA, was due in part to patients having a lower BMI at baseline. Importantly, the small sample size of 138 meeting the inclusion criteria restricted the size of the EA $(n=65)$ and no EA $(n=73)$ groups. Validation of the findings in a larger cohort is warranted. Future research in multicenter studies is required to fully elucidate the most effective criteria for EA implementation and the most appropriate nutritional supplements to improve clinical outcome of patients with ESCC undergoing trimodality treatment.
In conclusion, the findings of this study showed that patients with EC with malnutrition status undergoing neoadjuvant therapy with EA had an improved outcome at maintaining nutritional status, reduced severity and frequency of mucositis, and improved survival rate. EA is an effective intervention; however, it may increase anemia.

\section{References}

1 Lin W-C, Ding Y-F, Hsu H-L, Chang J-H, Yuan KS-P, Wu ATH, Chow J-M, Chang C-L, Chen S-U and Wu S-Y: Value and application of trimodality therapy or definitive concurrent chemoradiotherapy in thoracic esophageal squamous cell carcinoma. Cancer 123(20): 3904-3915, 2017.

2 Bower MR and Martin RC, 2nd: Nutritional management during neoadjuvant therapy for esophageal cancer. J Surg Oncol 100(1): 82-87, 2009.

3 Nozoe T, Kimura Y, Ishida M, Saeki H, Korenaga D and Sugimachi K: Correlation of pre-operative nutritional condition with post-operative complications in surgical treatment for oesophageal carcinoma. European Journal of Surgical Oncology (EJSO) 28(4): 396-400, 2002.

4 Bower M, Jones W, Vessels B, Scoggins C and Martin R: Nutritional support with endoluminal stenting during neoadjuvant therapy for esophageal malignancy. Ann Surg Oncol 16(11): 3161-3168, 2009.

5 Ben-David K, Kim T, Caban AM, Rossidis G, Rodriguez SS and Hochwald SN: Pre-therapy laparoscopic feeding jejunostomy is safe and effective in patients undergoing minimally invasive esophagectomy for cancer. J Gastroint Surg 17(8): 1352-1358, 2013.

6 Motoori M, Yano M, Yasuda T, Miyata H, Peng YF, Yamasaki M, Shiraishi O, Tanaka K, Ishikawa $\mathrm{O}$ and Shiozaki $\mathrm{H}$ : Relationship between immunological parameters and the severity of neutropenia and effect of enteral nutrition on immune status during neoadjuvant chemotherapy on patients with advanced esophageal cancer. Oncology 83(2): 91-100, 2012.

7 Cox S, Powell C, Carter B, Hurt C, Mukherjee S and Crosby TDL: Role of nutritional status and intervention in oesophageal cancer treated with definitive chemoradiotherapy: Outcomes from scope1. Br J C ancer 115(2): 172, 2016.

8 NCCN clinical practice guidelines in oncology (NCCN guidelines): Esophageal and esophagogastric junction cancer, version 3. (2015). Available at http://www.nccn.org/ professionals/physician_gls/pdf/esophageal.pdf.

9 Lordick F, Mariette C, Haustermans K, Obermannová R and Arnold D: Oesophageal cancer: Esmo clinical practice guidelines for diagnosis, treatment and follow-up. AnnOncol 27(suppl 5): v50-v57, 2016.

10 Cools-Lartigue J, Jones D, Spicer J, Zourikian T, Rousseau M, Eckert E, Alcindor T, Vanhuyse M, Asselah J and Ferri L: Management of dysphagia in esophageal adenocarcinoma patients undergoing neoadjuvant chemotherapy: Can invasive tube feeding be avoided? Ann Surg Oncol 22(6): 1858-1865, 2015.

11 Huerter ME, Charles EJ, Downs EA, Hu Y, Lau CL, Isbell JM, McMurry TL and Kozower BD: Enteral access is not required for esophageal cancer patients undergoing neoadjuvant therapy. Ann Thorac Surg 102(3): 948-954, 2016. 
12 Sunde B, Ericson J, Kumagai K, Lundell L, Tsai J, Lindblad M, Rouvelas I, Friesland S, Wang N and Nilsson M: Relief of dysphagia during neoadjuvant treatment for cancer of the esophagus or gastroesophageal junction. Dis Esophag 29(5): 442-447, 2016.

13 Jenkins TK, Lopez AN, Sarosi GA, Ben-David K and Thomas RM: Preoperative enteral access is not necessary prior to multimodality treatment of esophageal cancer. Surgery 163(4): 770-776, 2018.

14 Nomura M, Shitara K, Kodaira T, Hatooka S, Mizota A, Kondoh C, Yokota T, Takahari D, Ura T and Muro K: Prognostic impact of the 6th and 7th American Joint Committee on Cancer TNM staging systems on esophageal cancer patients treated with chemoradiotherapy. Int Radiat Oncol Biol Phys 82(2): 946-952, 2012.

15 Oken MM, Creech RH, Tormey DC, Horton J, Davis TE, McFadden ET and Carbone PP: Toxicity and response criteria of the Eastern Cooperative Oncology Group. Am J Clin Oncol 5(6): 649-655, 1982.

16 Miyazaki T, Sakai M, Sohda M, Tanaka N, Yokobori T, Motegi Y, Nakajima M, Fukuchi M, Kato H and Kuwano H: Prognostic significance of inflammatory and nutritional parameters in patients with esophageal cancer. Anticancer Res 36(12): $6557-$ 6562, 2016.

17 Birnstein E and Schattner M: Nutritional support in esophagogastric cancers. Surg Oncol Clin N Am 26(2): 325-333, 2017.

18 Aoyagi T, Terracina KP, Raza A, Matsubara H and Takabe K: Cancer cachexia, mechanism and treatment. World J Gastroint Oncol 7(4): 17, 2015.

19 Sultan J, Griffin SM, Di Franco F, Kirby JA, Shenton BK, Seal CJ, Davis P, Viswanath YK, Preston SR and Hayes N: Randomized clinical trial of omega-3 fatty acid-supplemented enteral nutrition versus standard enteral nutrition in patients undergoing oesophagogastric cancer surgery. Br J Surg 99(3): 346-355, 2012.

20 Miyata H, Yano M, Yasuda T, Hamano R, Yamasaki M, Hou E, Motoori M, Shiraishi O, Tanaka K, Mori M and Doki Y: Randomized study of clinical effect of enteral nutrition support during neoadjuvant chemotherapy on chemotherapy-related toxicity in patients with esophageal cancer. Clin Nutr 31(3): 330336, 2012.

21 Cools-Lartigue J, Jones D, Spicer J, Zourikian T, Rousseau M, Eckert E, Alcindor T, Vanhuyse M, Asselah J and Ferri LE: Management of dysphagia in esophageal adenocarcinoma patients undergoing neoadjuvant chemotherapy: Can invasive tube feeding be avoided? Ann Surg Oncol 22(6): 1858-1865, 2015.

22 Starr B, Davis S, Ayala-Peacock D, Blackstock WA and Levine EA: Reassessment of the role of enteral tube feedings for patients with esophageal cancer. Am Surgeon 80(8): 752-758, 2014

23 Lu C-L, Lang H-C, Luo J-C, Liu C-C, Lin H-C, Chang F-Y and Lee S-D: Increasing trend of the incidence of esophageal squamous cell carcinoma, but not adenocarcinoma, in Taiwan Cancer Causes Control 21(2): 269-274, 2010.
24 Vaughan TL, Davis S, Kristal A and Thomas DB: Obesity, alcohol, and tobacco as risk factors for cancers of the esophagus and gastric cardia: Adenocarcinoma versus squamous cell carcinoma. Cancer Epidemiol Prev Biomark 4(2): 85-92, 1995.

25 Hayashi Y, Correa AM, Hofstetter WL, Vaporciyan AA, Rice DC, Walsh GL, Mehran RJ, Lee JH, Bhutani MS and Dekovich A: The influence of high body mass index on the prognosis of patients with esophageal cancer after surgery as primary therapy. Cancer 116(24): 5619-5627, 2010.

26 Grotenhuis BA, Wijnhoven BPL, Hötte GJ, van der Stok EP, Tilanus HW and van Lanschot JJB: Prognostic value of body mass index on short-term and long-term outcome after resection of esophageal cancer. World J Surg 34(11): 2621-2627, 2010.

27 Keefe DM and Gibson RJ: Mucosal injury from targeted anticancer therapy. Supportive Care Cancer 15(5): 483-490, 2007.

28 Crosby T, Hurt CN, Falk S, Gollins S, Mukherjee S, Staffurth J, Ray R, Bashir N, Bridgewater JA and Geh JI: Chemoradiotherapy with or without cetuximab in patients with oesophageal cancer (scope1): A multicentre, phase 2/3 randomised trial. Lancet Oncol 14(7): 627-637, 2013.

29 Gwynne S, Hurt C, Evans M, Holden C, Vout L and Crosby T: Definitive chemoradiation for oesophageal cancer - a standard of care in patients with non-metastatic oesophageal cancer. Clin Oncol 23(3): 182-188, 2011.

30 Lv J, Cao X-F, Zhu B, Ji L, Tao L and Wang D-D: Long-term efficacy of perioperative chemoradiotherapy on esophageal squamous cell carcinoma. World J Gastroenterol 16(13): 16491654,2010

31 van Hagen P, de Jonge R, van Berge Henegouwen M, Hötte G, van der Stok E, Lindemans J, van Lanschot J and Wijnhoven B: Vitamin b12 deficiency after esophagectomy with gastric tube reconstruction for esophageal cancer. Dis Esophagus 30(12): 1$8,2017$.

32 Marimuthu K, Varadhan KK, Ljungqvist O and Lobo DN: A meta-analysis of the effect of combinations of immune modulating nutrients on outcome in patients undergoing major open gastrointestinal surgery. Ann Surg 255(6): 1060-1068, 2012.

33 Cheng Y, Zhang J, Zhang L, Wu J and Zhan Z: Enteral immunonutrition versus enteral nutrition for gastric cancer patients undergoing a total gastrectomy: A systematic review and meta-analysis. BMC Gastroenterol 18(1): 11, 2018.

34 Wong CS and Aly EH: The effects of enteral immunonutrition in upper gastrointestinal surgery: A systematic review and metaanalysis. Int J Surg 29: 137-150, 2016.
Received October 27, 2018

Revised November 9, 2018

Accepted November 13, 2018 